instrumenteller Natur, welche Belopolsky ${ }^{1}$ ) selbst hervorhebt, ungünstig beeinflußt.

Sonst liegen nur noch einzelne, gelegentliche Beobachtungen vor, und zwar von $W$. $W$. Campbell (Ap. J. 8. I 50, 14. I 4 I, Lick Bull. 1.23), Frost und Adams (Ap. J. 18.273), Lord (Ap. J. 2 I.3 I 4), Slipher (Ap. J. 22.328), Kiistner (Ap. J. 27.301) und Plaskett (Report of Chief-astronomer (Ottawa) I 906 und Journ. R. A. Soc. of Canada I. I 2 I l. Die Verwertung

Wien und Potsdam, 19 I 2 April. dieser einzelnen Reihen ist aber deswegen nicht möglich, weil sich ihre gegenseitigen systematischen Differenzen bei der kleinen Zahl der Beobachtungen nicht mit der erforderlichen Sicherheit ableiten lassen. Korrigiert man sie jedoch um das jeweilige Mittel ihrer Abweichungen von den Potsdamer Bestimmungen, so tritt ein Widerspruch zwischen ihnen und der oben abgeleiteten Geschwindigkeitskurve nicht hervor.

3) Mitt. Pulk. 3.209.

Adolf Hnatek.

Beobachtungen am $203 \mathrm{~mm}$-Refraktor der Breslauer Sternwarte.

$\mathrm{M}=$ L. Mewes, $\mathrm{P}=F$. Pavel, $\mathrm{B}=$ G. Brucks.

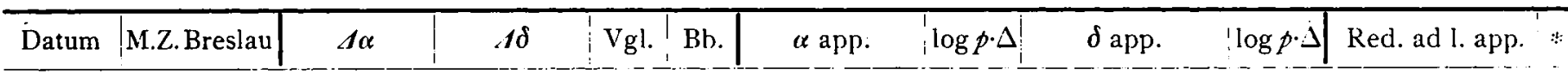

Borrellyscher Komet iqi I e.

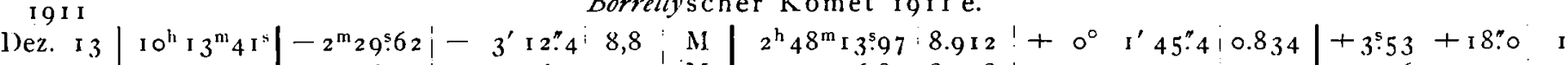

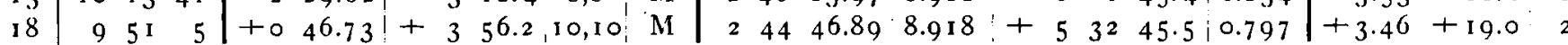

5 I Nemausa.

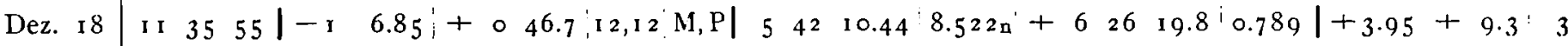
I9I 2 Ig 2 Nausikaa.

Febr. 8 I

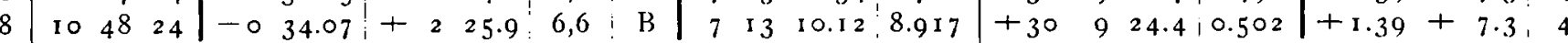

8

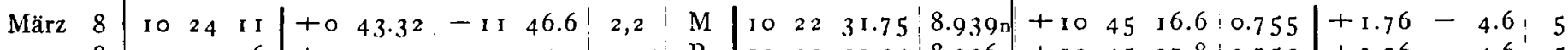

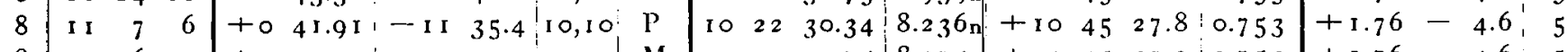

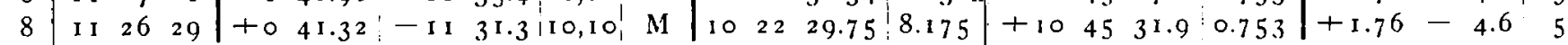

354 Eleonora.

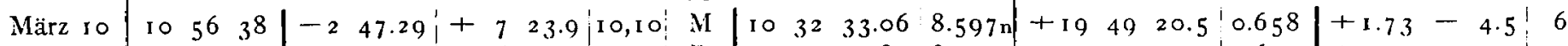

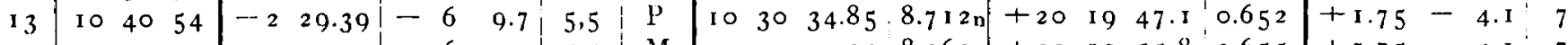

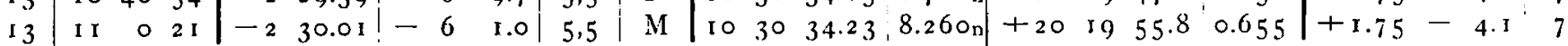
34 Circe.

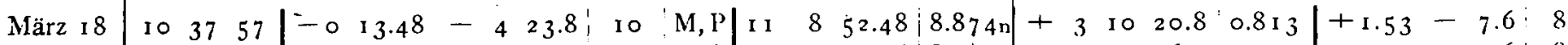

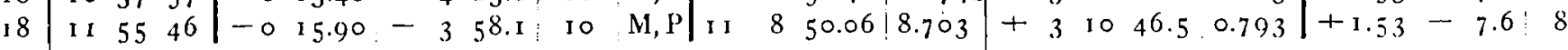

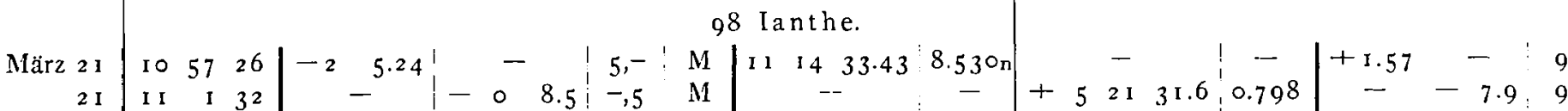

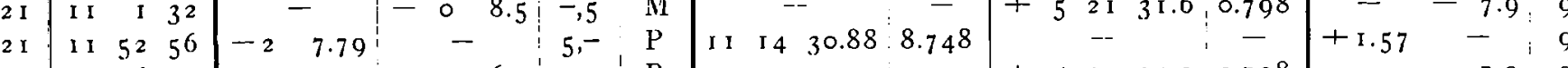

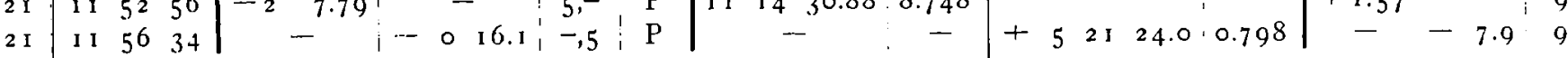
35 Leukothea.

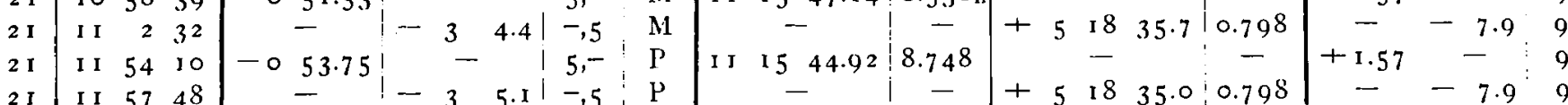

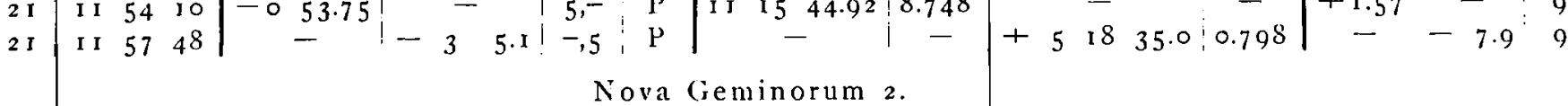

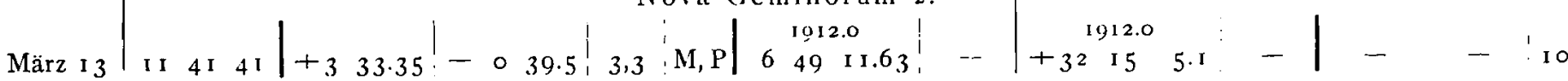

Die Beobachtungen wurden mit 2 I 5 facher Vergrößerung und bei ruhendem Fernrohr angestelit, 1 c wurde mit dem Chronographen, $1 \delta$. mit der Schraube gemessen. Nur die beiden Bestimmungen von 34 Circe wurden wegen der Nähe des Vergleichsternes durch Positionswinkel und Distanz erhalten. 
Mittlere Örter der Vergleichsterne.

\begin{tabular}{|c|c|c|c|}
\hline$*$ & $\alpha$ & $\delta$ & Autorität \\
\hline & I 9 I I.O & I 9 I I.O & \\
\hline I & $2^{\mathrm{h}} 50^{\mathrm{m}} 40^{\mathrm{s}} .06$ & $+0^{\circ} 4^{\prime} 39^{\prime \prime} .8$ & AG Nic 610 \\
\hline 2 & $\begin{array}{lll}2 & 43 & 56.70\end{array}$ & +52830.3 & AG Lpz II IO4 I \\
\hline 3 & $\begin{array}{c}543 \text { I } 3.34 \\
\text { I } 9 \text { I } 2.0\end{array}$ & $\begin{array}{c}+62523.8 \\
\text { I9 } 12.0\end{array}$ & $» \quad 24$ I I \\
\hline 4 & 7 I 342.80 & $+3065 \mathrm{I.2}$ & Oxfph Pl. 2 গOI Nr. 18487 \\
\hline
\end{tabular}

Breslau, I 9 I 2 April 17 .

\begin{tabular}{|c|c|c|c|}
\hline$*$ & $\alpha$ I9 2.0 & $\delta 1912.0$ & Autorität \\
\hline 5 & $I^{h^{h}}{ }_{2}{ }^{m_{4}} 46.67$ & $+10^{\circ} 57^{\prime} 7^{\prime \prime} 8$ & $A G L p z I 402 r$ \\
\hline 6 & io 35 I 8.62 & +1942 & $\mathrm{AG}$ Berl $\mathrm{A}_{4224}$ \\
\hline 7 & $1033 \quad 2.49$ & +2026 & AG Berl B 4068 \\
\hline 8 & $\begin{array}{lll}\text { I I } & 9 & 4.43\end{array}$ & +31452.2 & AG Alb 4222 \\
\hline 9 & II 1637.10 & $+52 \mathrm{I} 48.0$ & AG Lpz II 5776 \\
\hline IO & $\begin{array}{lll}6 & 45 & 38.28\end{array}$ & +32 I5 44.6 & AG Lei 2845 \\
\hline
\end{tabular}

L. Mewes.

\section{Petites planètes et comètes}

observées à l'Equatorial de ro pouces de l'Observatoire de Genève.

\begin{tabular}{l|l|l|l|l|l|l|l|l|l|l|}
\hline Date & T.m. Gen. & $L$ & $\alpha \delta$ & Cp. Obs. & $\alpha$ app. & Parall. & $\delta$ app. & Parall. & Red. ad l. app. & $*$ \\
\hline
\end{tabular}

I910 24 r Germania.

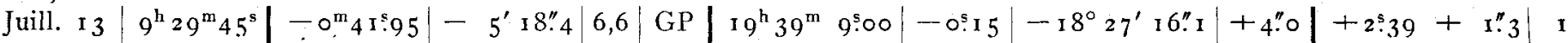

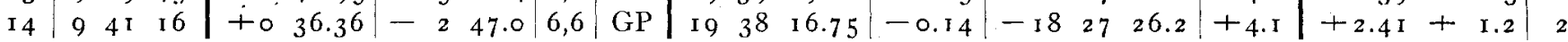

82 Alkmene.

Déc. $20 \mid \begin{array}{llll}6 & 4 & 28 & 8\end{array}$ + I 9 I I 704 Interamnia.

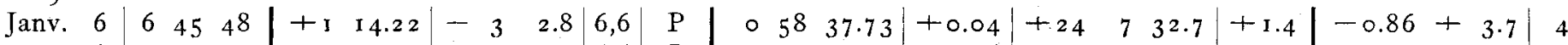

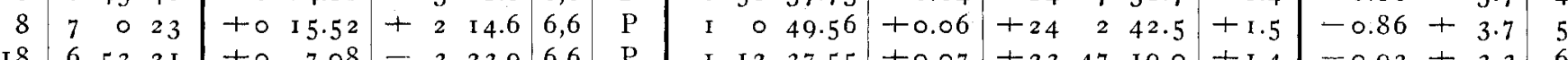

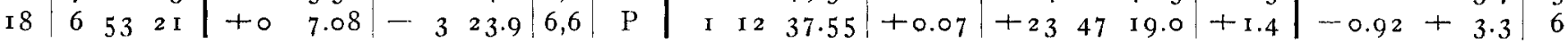

79 Eurynome.

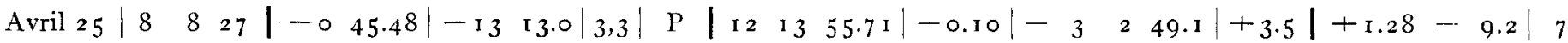
I 910

Comète de Faye rq io e.

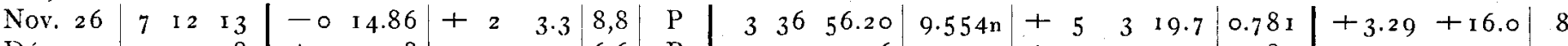

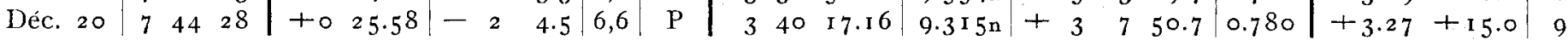
I 9 I I

Comète ig i c (Brooks).

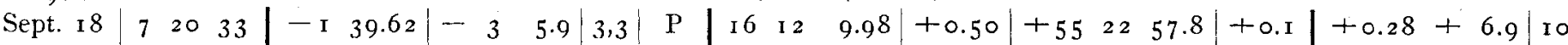

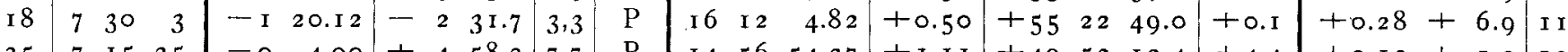

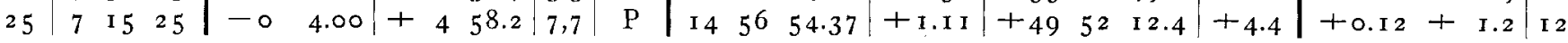

Comète r 9 i r f (Quenisset).

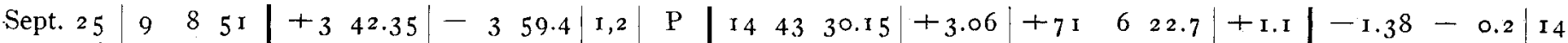

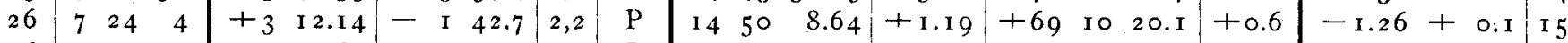

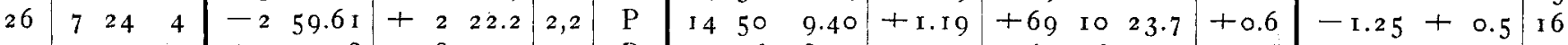

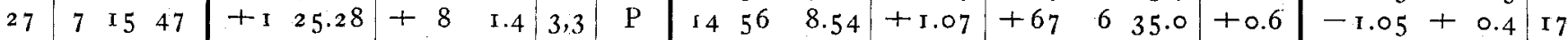

Oct. I 8 7 7 I 7 7

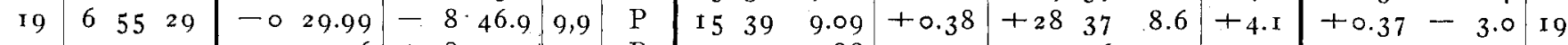
$20 \quad 74434+$ to $50.96+855.244$ P I $53950.88+0.39+27$ I6 $27.2+4.7+0.40-3.320$

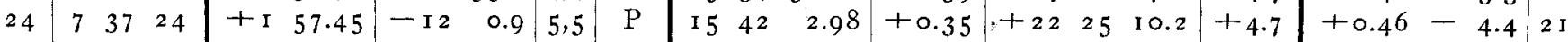
\begin{tabular}{l|lll|ll}
30 & 7 & I 2 & 53 & +2 & 4.32 \\
\hline
\end{tabular}

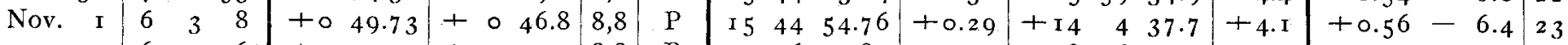
$7|69556|+$ I I $5.92|+041.0| 8,8 \mid$ P $\mid$ I $54_{4} 65.83|+0.27|+83642.3|+4.1|+0.64-7.7 \mid 24$

Positions moyennes des étoiles de comparaison référées au commencement de l'année d'observation.

\begin{tabular}{|c|c|c|c|c|c|c|c|}
\hline$*$ & $\alpha$ & $\delta$ & Autorité & $*$ & $\alpha$ & $\delta$ & Autorité \\
\hline I & $19^{\mathrm{h}} 39^{\mathrm{m}} 4^{8} \cdot 56$ & $-\mathrm{I} 8^{\circ} 2 \mathrm{I}^{\prime} 59^{\prime \prime} \circ$ & Bord $_{90} 5957$ & 3 & $4^{\mathrm{h}} 26^{\mathrm{m}} 19^{\mathrm{s}} \cdot 5^{2}$ & $+25^{\circ} 4 \mathrm{r}^{\prime} 34^{\prime \prime} 3$ & AG Cbr E. 2100 \\
\hline 2 & I9 $37 \quad 37.98$ & - I $8 \quad 24 \quad 40.4$ & $\gg \quad 5947$ & 4 & - $57 \quad 24.37$ & +24 10 31.8 & AG Berl B 3 I 5 \\
\hline
\end{tabular}

